\title{
Vitamin D related genes in lung development and asthma pathogenesis
}

\author{
Alvin T Kho 1,2, Sunita Sharma 2,3,4, Weiliang Qiu, ${ }^{3,8}$, Roger Gaedigk ${ }^{5,9}$, Barbara Klanderman ${ }^{3,8}$, Simin Niu $u^{3,8}$, \\ Chris Anderson ${ }^{7,10}$, James S Leeder ${ }^{5,9}$, Scott T Weiss ${ }^{2,3,6,8}$ and Kelan G Tantisira $2,3,4,8^{*}$
}

\begin{abstract}
Background: Poor maternal vitamin D intake is a risk factor for subsequent childhood asthma, suggesting that in utero changes related to vitamin $\mathrm{D}$ responsive genes might play a crucial role in later disease susceptibility. We hypothesized that vitamin D pathway genes are developmentally active in the fetal lung and that these developmental genes would be associated with asthma susceptibility and regulation in asthma.
\end{abstract}

Methods: Vitamin D pathway genes were derived from PubMed and Gene Ontology surveys. Principal component analysis was used to identify characteristic lung development genes.

Results: Vitamin D regulated genes were markedly over-represented in normal human (odds ratio OR 2.15, 95\% confidence interval Cl: 1.69-2.74) and mouse (OR 2.68, 95\% Cl: 2.12-3.39) developing lung transcriptomes. 38 vitamin D pathway genes were in both developing lung transcriptomes with $>63 \%$ of genes more highly expressed in the later than earlier stages of development. In immortalized B-cells derived from 95 asthmatics and their unaffected siblings, 12 of the $38(31.6 \%)$ vitamin D pathway lung development genes were significantly differentially expressed (OR 3.00, 95\% Cl: 1.43-6.21), whereas 11 (29\%) genes were significantly differentially expressed in 43 control versus vitamin D treated immortalized B-cells from Childhood Asthma Management Program subjects (OR 2.62, 95\% Cl: 1.22-5.50). 4 genes, LAMP3, PIP5K1B, SCARB2 and TXNIP were identified in both groups; each displays significant biologic plausibility for a role in asthma.

Conclusions: Our findings demonstrate a significant association between early lung development and asthmarelated phenotypes for vitamin D pathway genes, supporting a genomic mechanistic basis for the epidemiologic observations relating maternal vitamin D intake and childhood asthma susceptibility.

Keywords: Vitamin D, Cholecalciferol, Lung development, Asthma, Fetal programming

\section{Background}

The prevalence of asthma, a disease affecting 300 million individuals worldwide [1], has risen substantially over the past 30 years. From an epidemiologic perspective, the increase in asthma has been greatest in industrialized countries and in those countries undergoing rapid urbanization $[2,3]$. This has led to the so-called Western lifestyles hypothesis, which states that factors accompanying the transition from a predominantly rural to a predominantly urban lifestyle may increase susceptibility to asthma and other auto-immune diseases [2-5]. One

\footnotetext{
* Correspondence: kelan.tantisira@channing.harvard.edu

${ }^{2}$ Harvard Medical School, Boston, MA, USA

${ }^{3}$ Channing Division of Network Medicine, Brigham and Women's Hospital,

181 Longwood Avenue, Boston, MA 02115, USA

Full list of author information is available at the end of the article
}

such factor is vitamin D deficiency. It has been estimated that one billion individuals worldwide are insufficient or deficient in vitamin D [6]. In Westernized countries, vitamin $\mathrm{D}$ levels tend to be low due to both increased urban (indoor) lifestyles and high use of sunscreen.

Multiple studies have now demonstrated an association between lower maternal vitamin $\mathrm{D}$ level during pregnancy and subsequent increased risk of childhood wheezing or asthma. For example, in an analysis of 1,194 mother-child pairs from Boston, MA, 3-year-old children born to mothers with vitamin D intake in the highest quartile during pregnancy had a $62 \%$ reduction in risk of recurrent wheeze (adjusted odds ratio OR 0.38, 95\% confidence interval CI: 0.22-0.65) [7]. Similar analyses of 1,212 and 763 mother-child pairs from Scotland

\section{Biomed Central}


and Japan showed a $67 \%$ and $36 \%$ reduction in risk for subsequent persistent wheeze for children with maternal vitamin $\mathrm{D}$ intake in the highest quintile [8,9]. Overall, high maternal dietary vitamin $\mathrm{D}$ intake during pregnancy appears to be protective for the development of wheezing outcomes (OR 0.56, 95\% CI: 0.42-0.73) [10].

The fetal origins or Barker [11] hypothesis states that in utero exposure to the maternal environment, including diet, may influence the eventual development of chronic disease. Globally, vitamin D has been postulated to be important in early pregnancy, regulating key target genes associated with implantation and implantation tolerance [12]. Vitamin D also regulates genes involved in the inflammation, immunity, cellular proliferation, and apoptosis associated with obstructive airways disease [13], likely via an epigenetic mechanism. Given the potential role that vitamin D plays in pregnancy, as well as the influence of maternal diet on subsequent childhood respiratory outcomes, it has been postulated that vitamin $D$ deficiency directly affects programming within the developing fetal lung in a manner that may influence disease susceptibility $[14,15]$. We hypothesized that vitamin D pathway genes are transcriptionally active and temporally regulated during normal fetal lung development. Given the association of maternal vitamin D intake to subsequent childhood asthma, we further hypothesized that a significant subset of vitamin $\mathrm{D}$ genes important to normal fetal lung development would also be asthma susceptibility genes. We tested this hypothesis through an integrative analysis of developing mouse and human fetal lung transcriptomes. We identified key vitamin D pathway lung development genes and tested their transcriptomic association with asthma susceptibility.

\section{Methods}

Derivation of the vitamin $D$ related gene set (VDRGS)

We assembled genes associated with vitamin D using both supervised and unsupervised approaches. In the supervised approach, we used genes recorded to be associated with vitamin $\mathrm{D}$ structure, function, regulation and signaling in Gene Ontology (GO, http://www.geneontology.org/ version May 2013) [16] or Entrez Gene (http:// www.ncbi.nlm.nih.gov/gene version May 2013) databases. In the unsupervised approach, we used 212 unique human (195 homologous mouse) genes reported to be differentially regulated post vitamin D stimulation in human lymphoblastoid B cell lines [17].

\section{Microarray data}

We used 3 developing lung time series datasets. The first is an expansion of the National Center for Biotechnology Information Gene Expression Omnibus (GEO, http:// www.ncbi.nlm.nih.gov/geo/) GSE11539 [18] of C57BL6 mouse whole lung at embryonic days 9.5 (E9.5), 12.5,
$14.5,16.5,18.5$, and postnatal days 0 (P0), 2, 4, 7, 11, 13, $18,24,30,56$ in biological duplicates profiled on Affymetrix Mouse Gene 1.0 ST array courtesy of Carol J. Bult of the Jackson Laboratory, Bar Harbor, ME. The study protocol was approved by the Jackson Laboratory Animal Care and Use Committee \#01011. The second GSE14334 consists of 38 human fetal lung samples from 53 to 154 estimated days post conception (dpc) profiled on the Affymetrix Human Genome U133 Plus 2.0 array [19]. The third GSE20954 consists of developing mouse whole lungs from E12 to P30 in duplicates profiled on Affymetrix Mouse Genome 430 Plus 2.0 array [20].

For asthma and vitamin D transcriptomic association analyses, we used 3 datasets: First, GSE8052 [21] consists of Epstein-Barr virus (EBV) transformed lymphoblastoid B-cells (LCLs) from a pediatric asthma family association study on the Affymetrix Human Genome U133 Plus 2.0 array. We restricted the data to 95 unaffected-affected sibling pairs (see Additional file 1: Table S1 and Figure S1). Second, we used LCLs from 43 asthmatic subjects from an ancillary genetics study of the Childhood Asthma Management Program (CAMP) $[22,23]$ that was approved by the Brigham and Women's Hospital Institutional Review Board \# 1999-P-001549. All subjects or their legal guardians provided written informed consent to participate in the study protocols. LCLs were cultured in RPMI-1640 supplemented with 5\% FBS and 1X Penicillin/Streptomycin/L-Glutamineto an average density of 200,000 cells/ $\mathrm{ml}$. The cells were then split and stimulated with sham (control) versus $1 \mu \mathrm{M}$ 1,25-OH vitamin $\mathrm{D}$ for 72 hours. Total RNA was extracted from the cells using the Absolutely RNA Miniprep column purification system (Stratagene, LaJolla, CA) per manufacturer's instructions. Paired RNA samples were profiled on the Illumina HumanHT-12 V4 array. Third, GSE5145 consists of vitamin $\mathrm{D}$ versus sham stimulated normal human bronchial smooth muscle cells [24].

For each developmental dataset and the first asthma dataset, sample files were processed using the Robust Multi-array Analysis (RMA) quantile normalization [25] package in BioConductor (http://www.bioconductor.org/) to produce an $\mathrm{N} \times \mathrm{M}$ data matrix of RMA signals in the logarithmic base $2(\log 2)$ scale. $\mathrm{N}$ is the total number of probe sets (genes) in the microarray platform and $M$ is the total number of samples. The second asthma dataset was further processed using the probe-wise nonparametric regression function locfit in BioConductor [26] to minimize the effects of subject or age estimation-related variation in expression measurement and to model global gene-specific developmental expression patterns. For each probe set in a developmental dataset, we computed the linear correlation between replicate time series expression profiles to assess the reproducibility of its sample expression profile. When a gene (its 
Entrez Gene ID) is represented by $>1$ probe sets, we selected the probe set with the maximum linear correlation between replicate to be its unique representative.

In the asthma datasets, Wilcoxon signed rank test was used to determine differential expression between the paired samples, i.e., affected versus unaffected sibling, preversus post- vitamin $D$ per subject at $p<0.05$ significance threshold. When a gene (its Entrez Gene ID) is represented by $>1$ probe sets, we selected the probe set with the smallest signed rank $\mathrm{p}$ value as its unique representative.

Principal component analysis for identifying characteristic genes in a developing organ transcriptome

Each developmental dataset is a $\mathrm{N}$ genes $\times \mathrm{M}$ samples data matrix of RMA signals. First, the columns of the data matrix were standardized to average 0 and variance 1 since we were investigating sample variation in the sense of linear correlation, as opposed to Euclidean distance. Second, we performed principal component analysis (PCA) of sample time points in gene space, and obtained the loading coefficients for each gene in principal components 1 to 3 (PC1-3) [19,27-29]. Each principal component (PC) is a linear combination of $\mathrm{N}$ genes. The magnitude of the loading coefficient of a gene in a given PC corresponds to its contribution to the sample variation along that PC. For each $\mathrm{PC}$, we ranked the genes in decreasing order of its loading coefficient magnitude. For example, the mouse gene $\mathrm{H} 2-\mathrm{Ab} 1$ ranked 42 in $\mathrm{PC} 2$ in the developing mouse lung dataset has the 42-nd highest loading coefficient magnitude in $\mathrm{PC} 2$. We define the characteristic genes for a PC to be the top 5\% ranking genes in that PC. A gene that is characteristic in any one of the $\mathrm{PC} 1-3$ in a given dataset is defined to be a characteristic gene for that dataset.

\section{Fold change, bio-ontology enrichment and overlap} analyses

The microarray reported gene expression intensity is an RMA signal in log2 scale. Suppose that each sample in a microarray dataset has a mutually exclusive condition label - A or B. The log2 fold change of a gene in A relative to $\mathrm{B}$ is the arithmetic average in A minus the arithmetic average in B. Fisher exact test on DAVID 6.7 http://david.abcc.ncifcrf.gov/ [30] was used to determine specific bio-ontological enrichment in a given gene set relative to a background gene set at $\mathrm{p}<0.05$ significance threshold. More generally, two-sided Fisher exact test was used to assess the significance of overlaps between any pair of gene sets, and a 95\% confidence interval (CI) was supplied for the odds ratio (OR).

\section{Results}

The vitamin $D$ related gene set (VDRGS)

For the supervised analysis, from GO, we found $24 \mathrm{GO}$ terms that contain character strings "vitamin $D^{*}$ " or "cholecalciferol*", where * denotes a wildcard character string. For example, the GO term "GO:0070561 vitamin $D$ receptor signaling pathway" contains 4 unique human genes: CYP24A1, CYP27B1, TRIM24 and VDR. The union of these $24 \mathrm{GO}$ terms contained 67 unique human genes. Similarly, Entrez Gene had 201 unique human genes with a description field containing "vitamin $\mathrm{D}^{* \text { " }}$ or "cholecalciferol":" The supervised approach from GO and Entrez Gene combined yielded 211 unique human (215 homologous mouse) genes, see Figure 1 and Additional file 1: Table S1. In the unsupervised approach, as noted, we assembled 212 previously reported unique human (195 homologous mouse) genes resulting from differential expression following vitamin D stimulation [17], see Figure 1 and Additional file 1: Table S1. 10 genes were common to the sets of genes derived using supervised and unsupervised approaches: CAMP, CD274, CYP19A1, CYP24A1, DHCR7, LGMN, MED13, NFKBIA, TNFSF4 and VDR. Together, the supervised and unsupervised approaches yielded 413 unique human (400 homologous mouse) genes that we define to be the vitamin $\mathrm{D}$ related gene set (VDRGS) in this study (Additional file 1: Table S1).

\section{Derivation of developing lung characteristic gene sets (DLCGS)}

Here we identify the main contributor genes to transcriptome scale sample variation in the developing whole fetal lung using principal component analysis (PCA) following our previous work [19,28], see Materials and Methods. We used 2 independent developing whole lung transcriptome

\section{VDRGS}

\section{Supervised Unsupervised}

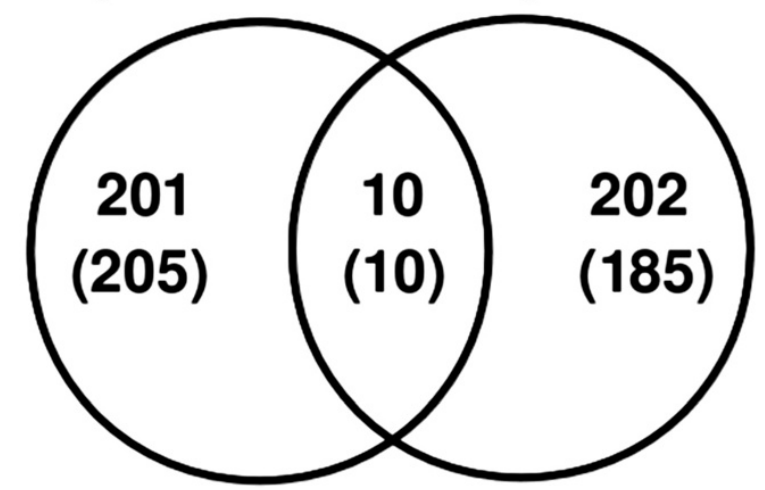

Figure 1 Composition of the vitamin D related gene set (VDRGS). Venn diagram of vitamin D related human gene sets assembled using supervised (GO and Entrez Gene databases) and unsupervised [17] approaches. Bracketed numbers (\#) refer to number of homologous mouse genes. 
time series: The C57BL6 mouse time series (E9.5 to P56) spanned the major histo-morphological stages of lung development: pseudoglandular, canalicular, saccular and alveolar, GSE11539 expanded [18]. The human time series (53 to $154 \mathrm{dpc}$ ) spanned the pseudoglandular and canalicular stages, GSE14334 [19].

For each time series, we applied PCA of sample time points in transcriptome space. In each case we observed that the sample configuration along the first three principal components (PC1-3) correlated with age, time-tobirth or a transition between histo-morphological stages of lung development. The loading coefficient magnitude of a gene in a given principal component is proportional to its contribution to the sample variation along that principal component, see Materials and Methods. Therefore we define the genes with the top 5\% highest loading coefficient magnitude in any one of PC1-3 to be the developing lung characteristic gene set (DLCGS) for the particular time series. These DLCGS consisted of 2,472 (human) and 2,495 (mouse) genes respectively. We had previously shown that these DLCGS were enriched for ontological attributes associated with developmental processes in general and the developing lung structure and function in particular [19,28] suggesting the DLCGS qualitatively capture developing lung biology.

\section{Over-representation of vitamin D related genes (VDRGS) in developing lung characteristic gene sets (DLCGS)}

We observed significant overlaps between the VDRGS with both DLCGS above: 103 VDRGS genes in the C57BL6 mouse DLCGS expanded GSE11439 (OR 2.68, 95\% CI: 2.12-3.39), and 92 VDRGS genes in the human DLCGS GSE14334 (OR 2.15, 95\% CI: 1.69-2.74) (Additional file 1: Figure S1). 38 VDRGS genes were common to both mouse and human DLCGS (Figure 2). The VDRGS was similarly found to overlap significantly with the DLCGS of an independent developing mouse lung time series (E12 to P30), GSE20954 (OR 2.07, 95\% CI: 1.60-2.68) [20].

Of the 38 common VDRGS-DLCGS genes, 29 (76\%) were expressed at a higher level in the later rather than earlier stages of the developing mouse lung; whereas 24 (63\%) were expressed at a higher level in the later canalicular than earlier pseudoglandular stages of the developing human lung (Figure 2).

\section{Profile of 38 common VDRGS-DLCGS genes in independ- ent pediatric asthma studies}

Here we investigate the 38 VDRGS genes common to both the C57BL6 mouse and human DLCGS in independent studies of the pediatric lymphoblastoid B-cell (LCL) transcriptome in unaffected versus affected asthma sibling pairs [21] and vitamin D versus control treatment of LCLs from asthmatic subjects from the Childhood Asthma Management Program (CAMP) [31] (Table 1).
For the pediatric asthma sibling pair study [21], 2,697 genes (of 20,188 unique genes measured) were differentially expressed between LCLs of 95 affected versus unaffected siblings pairs at $\mathrm{p}<0.05$. There was a significant overlap of 86 genes between this 2,697-gene set and the VDRGS (OR 1.77, 95\% CI: 1.38-2.27). Focusing on the 38 common VDRGS-DLCGS genes, 12 (31\%) were significantly differentially expressed between unaffectedaffected siblings: CD44, HLA-DRB1, IFIH1, IGF1*, LAMP3, LY75, MYC, PIP5K1B*, SCARB2*, SFRP1, TXNIP* and TYMS*, where* indicates under expression in the affected relative to the unaffected sibling pair (OR 3.00, 95\% CI: 1.43-6.21) (Figure 3).

For the vitamin D treatment study of 43 asthmatic CAMP subjects, 3,368 genes (of 24,960 unique genes measured) were differentially expressed between LCLs of vitamin $\mathrm{D}$ versus control treatment at $\mathrm{p}<0.05$. There was a significant overlap of 161 genes between this 3,368-gene set and the VDRGS (OR 4.30, 95\% CI: 3.505.29) (Figure 3). Focusing on the 38 common VDRGSDLCGS genes, 11 (22\%) were significantly differentially expressed between control-vitamin D treatment: $C A V 1$ *, CD69, IF144*, LAMP3, NAPSA, PIP5K1B, PRNP, SAT1, $S C A R B 2 *$ TXNIP* and VASH2, where* indicates underexpression in vitamin $\mathrm{D}$ relative to control treatment (OR 2.62, 95\% CI: 1.22-5.50) (Table 1). Four VDRGSDLCGS genes were common to both asthma/VDRGSDLCGS analyses above: LAMP3, PIP5K1B2, SCARB2 and TXNIP. Interestingly, the SNP rs975645 of PIP5K1B2 was found to be associated with asthma under a dominant genetic model in our previous CAMP study $(\mathrm{p}=0.004)$ [32].

In an unrelated study of vitamin D stimulated normal human bronchial smooth muscle cells GSE5145 [24], of the 405 genes that were 2-fold differentially expressed between vitamin $\mathrm{D}$ versus control treatment, we found a significant overlap of 9 genes with the VDRGS (OR 5.09, 95\% CI: 2.38-10.51) (Figure 3). Of these 9 genes, INSIG1 was also in VDRGS-DLCGS.

\section{Discussion}

We have performed a multi-staged analysis that demonstrates the prominence of vitamin D within the developing lung transcriptome and that supports the role of these developmental genes in asthma pathogenesis. Specifically, through interrogation of both supervised (literature based) and unsupervised (ChIP-Seq based) lists of vitamin $\mathrm{D}$ pathway genes, we have demonstrated that a significant proportion of vitamin D response elements are transcriptomically active during both normal human and mouse lung development. These vitamin D genes generally increase in expression throughout fetal lung development, with peak expression just prior to birth. We subsequently have shown that approximately $1 / 3$ (12 of 38) of the vitamin D genes that jointly influence both 


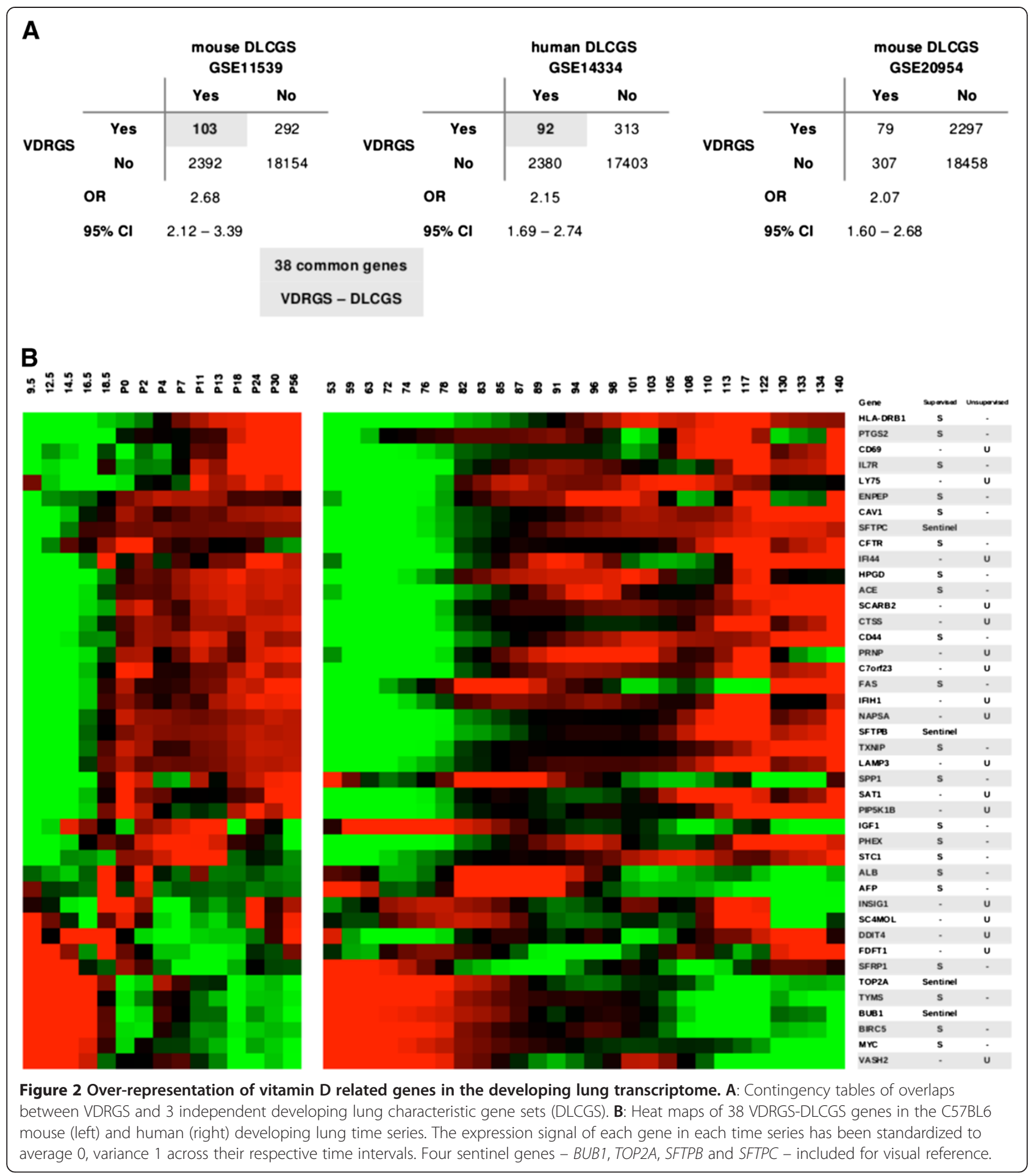

human and murine lung development are also differentially expressed in LCLs derived from asthmatic children as compared to non-asthmatic sibling controls. Overall, vitamin D lung developmental genes were three times as likely to be differentially expressed in these asthmatics, when compared to non-vitamin D genes. Finally, we noted that, of the 12 vitamin D lung developmental genes transcriptomically related to asthma susceptibility, 4 - LAMP1, PIP5K1B, SCARB2, and TXNIP - were significantly differentially expressed upon administration of vitamin $\mathrm{D}$ to cells derived from asthmatic children. Thus, multiple common vitamin $\mathrm{D}$ response elements appear to be important in both the developing lung and asthma, thereby providing a genomic rationale 
Table 138 Vitamin D regulated lung developmental genes and their log2 fold changes in 3 asthma and vitamin D stimulation studies: Lymphoblastoid B-cells of pediatric asthma (GSE8052) and vitamin D treatment (CAMP 43), and normal human bronchial smooth muscle vitamin D treatment (GSE5145)

\begin{tabular}{|c|c|c|c|c|c|c|}
\hline Gene ID & Gene & Supervised & Unsupervised & GSE8052 asthma/unaffected & CAMP 43 vitamin D/control & GSE5145 vitamin D/control \\
\hline 174 & AFP & S & - & 0.007 & -0.047 & 0.094 \\
\hline 213 & ALB & S & - & -0.023 & 0.074 & -0.130 \\
\hline 332 & $\mathrm{BIRC5}$ & S & - & -0.133 & -0.030 & 0.055 \\
\hline 355 & FAS & S & - & 0.098 & 0.060 & 0.189 \\
\hline 857 & CAV1 & s & - & -0.058 & -0.169 & 0.141 \\
\hline 950 & SCARB2 & - & $U$ & -0.341 & -0.124 & -0.174 \\
\hline 960 & CD44 & S & - & 0.155 & 0.107 & 0.325 \\
\hline 969 & CD69 & - & $U$ & 0.046 & 0.239 & 0.022 \\
\hline 1080 & CFTR & S & - & 0.022 & -0.103 & 0.184 \\
\hline 1520 & CTSS & - & $U$ & 0.053 & 0.026 & 0.301 \\
\hline 1636 & ACE & S & - & 0.062 & -0.148 & -0.262 \\
\hline 2028 & ENPEP & S & - & 0.025 & 0.047 & 0.651 \\
\hline 2222 & FDFT1 & - & $U$ & 0.080 & 0.056 & 0.125 \\
\hline 3123 & HLA-DRB1 & S & - & 0.130 & -0.090 & -0.107 \\
\hline 3248 & HPGD & S & - & 0.022 & -0.040 & -0.134 \\
\hline 3479 & IGF1 & S & - & -0.094 & 0.071 & 0.436 \\
\hline 3575 & IL7R & S & - & 0.027 & 0.075 & 0.310 \\
\hline 3638 & INSIG1 & - & $U$ & -0.025 & 0.065 & 1.223 \\
\hline 4065 & LY75 & - & $U$ & 0.208 & 0.108 & -0.163 \\
\hline 4609 & MYC & S & - & 0.124 & 0.124 & -0.239 \\
\hline 5251 & PHEX & S & - & 0.005 & 0.031 & -0.084 \\
\hline 5621 & PRNP & - & $U$ & 0.036 & 0.101 & 0.362 \\
\hline 0.362 & PTGS2 & S & - & 0.013 & 0.087 & 0.044 \\
\hline 6303 & SAT1 & - & $U$ & 0.077 & 0.141 & 0.064 \\
\hline 6307 & SC4MOL & - & $U$ & 0.070 & 0.058 & 0.297 \\
\hline 6422 & SFRP1 & S & - & 0.084 & 0.054 & -0.335 \\
\hline 6696 & SPP1 & S & - & -0.002 & -0.073 & -0.035 \\
\hline 6781 & STC1 & S & - & -0.052 & -0.085 & -0.401 \\
\hline 7298 & TYMS & S & - & -0.062 & 0.032 & -0.115 \\
\hline 8395 & PIP5K1B & - & U & -0.239 & 0.149 & 0.107 \\
\hline 9476 & NAPSA & - & $U$ & -0.032 & 0.388 & 0.036 \\
\hline 10561 & IFI44 & - & $U$ & -0.183 & -0.098 & -0.326 \\
\hline 10628 & TXNIP & S & - & -0.211 & -0.360 & -0.151 \\
\hline 27074 & LAMP3 & - & $U$ & 0.127 & 0.080 & 0.097 \\
\hline 54541 & DDIT4 & - & U & 0.080 & -0.018 & 0.456 \\
\hline 64135 & $\mathrm{IF|H} 1$ & - & $U$ & 0.178 & 0.058 & -0.243 \\
\hline 79161 & TMEM243 & - & U & 0.000 & 0.054 & 0.168 \\
\hline 79805 & VASH2 & - & U & 0.090 & 0.302 & 0.179 \\
\hline
\end{tabular}

Bold log2 values indicate statistical significance.

as a basis for the influence of maternal diet on later asthma susceptibility.

This work lends credence to the evolving literature surrounding the developmental origins hypothesis of complex disease pathogenesis. Prior work have established that dietary changes, including protein and caloric restriction, as well as environmental exposures in pregnant animals can result in changes in gene expression in 

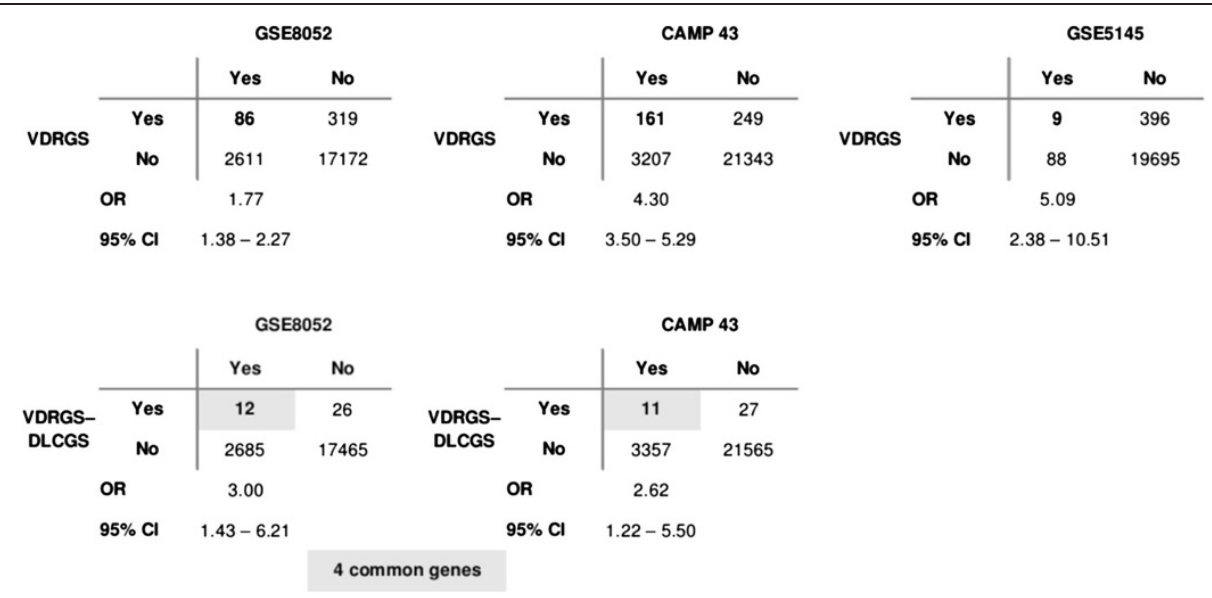

Figure 3 Over-representation of vitamin D genes in 3 asthma and vitamin D stimulation studies. Contingency tables of overlaps between VDRGS (and 38 VDRGS-DLCGS) and significantly differentially expressed genes in lymphoblastoid B-cells of pediatric asthma (GSE8052) and vitamin D treatment (CAMP 43), and normal human bronchial smooth muscle vitamin D treatment (GSE5145) studies.

the neonate that persist into adulthood [33-36]. Moreover, in humans, maternal vitamin D levels affect both placental calcium transport and bone mass in later life. These effects are thought to be mediated by vitamin $\mathrm{D}$ induction of the PMCA (plasma membrane $\mathrm{Ca}^{2+}$ ATPase) gene $[8,37]$. While the current study was not designed to demonstrate a direct correlation between maternal vitamin D status and neonatal outcomes, it does clearly indicate that vitamin $\mathrm{D}$ regulated processes are a part of the normal fetal lung developmental process. Combining this with the strong epidemiologic evidence linking maternal vitamin D status during pregnancy to subsequent asthma, our data suggest that maternal vitamin $\mathrm{D}$ insufficiency may lead to differential developmental regulation of key vitamin $\mathrm{D}$ genes within the fetal lung and thus, increase risk of childhood asthma. This hypothesis is further supported by the differences in expression of a significant number of developmental vitamin D genes in siblings discordant for asthma.

Our data further suggest that a subset of the lung developmental vitamin $\mathrm{D}$ asthma genes continue to be actively regulated in later life by vitamin $\mathrm{D}$, and thus, may continue to modify the asthma phenotype. The 4 genes that demonstrate this phenomenon in immortalized B cells derived directly from persistent childhood asthmatics likely represent a subset of vitamin D pathway genes involved in the immune mediation of asthma. Given their fetal lung and subsequent immune cell localization, it is possible that the remainder of the developmental 12 vitamin D genes that are differentially expressed in asthma may influence asthma in cells specific to the lung, such as epithelial or airway smooth muscle cells.

As mentioned, 4 of the developmental genes: LAMP1, PIP5K1B, SCARB2 and TXNIP, were differentially expressed in both asthma and upon stimulation of immortalized B-cells derived from asthmatics, suggesting a possible role of these genes in modulating the immune response in asthma. Interestingly, although these genes have generally not been implicated in allergic airways disease, each of these genes may be related to asthma pathogenesis via a distinct molecular mechanism. TXNIP, also known as vitamin D3 up-regulated protein 1 , is required for the development of natural killer cells [38]. In turn, CD4+Va24+ natural killer cells are significantly decreased in association with infection-associated asthma exacerbations and sputum eosinophil counts [39]. Notably, our microarray association demonstrated decreased expression of TXNIP in asthma; this association has been independently validated in a comparison of active asthmatics vs. normal volunteers [40].

While the other three genes have not been associated with asthma, they provide potentially interesting insights into the diversity of vitamin D biology. LAMP3 appears to be a marker of dendritic cell maturation [41] and has been implicated in the pathogenesis of psoriasis vulgaris [42]. PIP5K1B regulates calcium signaling in mast cells [43]. Both the dendritic cell and the mast cell are key regulators in the initiation of the asthmatic inflammatory response. Furthermore, the SNP rs975645 of PIP5K1B2 was found to be associated with asthma under a dominant genetic model in our previous CAMP study ( $\mathrm{p}=$ 0.004) [32]. In lieu of direct actions on inflammatory cells, $S C A R 2 B$ appears to modulate the normal maturation of phagosomes and autophagosomes $[44,45]$. While the exact role of autophagy in asthma remains to be determined, it has been hypothesized that autophagy is likely an important modulator of the lack of viral-induced apoptosis noted in subjects with asthma [46]. Consistent with this, a recent brief report noted the presence of 
autophagosomes in the airways of a subject with asthma; a comparable tissue from a control subject failed to detect any autophagosomes [47].

There are several limitations to our data. Our human lung samples were obtained from healthy aborted fetuses and thus inherently limited in their gestational age range to $\leq 20$ estimated weeks. While the vitamin D pathway appears to be active in early fetal development [12], it is unclear if perturbations in early or late gestation are most salient to asthma susceptibility. For this reason, we intersected our human developmental vitamin D list with a murine developmental dataset in which later developmental time periods were represented. Our sampling technique also precludes us from specifically ascertaining the direct effect of maternal vitamin D levels on the developing human lung. Instead, we focused on a combined approach that focused on the identification of significant vitamin $\mathrm{D}$ regulatory genes from the literature and a carefully performed ChIP-Seq study of the vitamin D receptor [17]. Finally, our asthma expression dataset analyses were performed in immortalized B-cells derived from asthmatics. While this may not specifically generalize the findings of the fetal lung expression datasets, the B-cell is a relevant asthma target cell [48-50] supporting the validity of these analyses. Only a small number of genes are specifically affected by the immortalization process [51]; none of these were among our reported vitamin D genes. Moreover, we have been able to previously correlate expression response of these genes to clinical asthma outcomes [31], further supporting the validity of our approach.

\section{Conclusions}

In conclusion, we have demonstrated that vitamin D genes are actively regulated in the developing human fetal lung and that a disproportionate number of these genes are differentially regulated in asthma. Not only does our study provide a mechanistic basis that helps to explain the developmental associations of vitamin D with asthma, it may also provide a methodologic blueprint for rationally interrogating diverse developmental pathways for their genomic association with subsequent disease outcomes.

\section{Additional file}

Additional file 1: Table S1. 413 vitamin D related genes. Figure S1. Heat maps of 103 and 92 vitamin D related lung genes overlapping with the developing lung characteristic genes of C57BL6 mouse (A) and human (B) developing lung time series respectively. The expression signal of each gene in each time series has been standardized to average 0 , variance 1 across their respective time intervals. Four sentinel genes $B U B 1$, TOP2A, SFTPB and SFTPC - included for visual reference.

\section{Abbreviations}

ChIP-Seq: Chromatin immunoprecipation with massively parallel DNA sequencing; DLCGS: Developing lung characteristic gene set; GO: Gene
Ontology; ID: Identifier; Log2: Logarithm base 2; PC\#: \#-th principal component; PCA: Principal component analysis; RMA: Robust multi-array analysis; VDRGS: Vitamin D related gene set.

\section{Competing interests}

The authors declare that they have no competing interests.

\section{Authors' contributions}

ATK contributed to the design, data analysis and writing. SS contributed to the data collection, analysis and writing. WQ, RG, BK, SM, CA, JSL contributed to the data collection and writing. STW contributed to the design and writing. KTG contributed to the design, analysis and writing. All authors read and approved the final manuscript.

\section{Acknowledgements}

This study was funded by NIH R01 HL097144, R01 HL092197, R21 HL107927 and U01 HL065899. ATK is supported by NIH K25 HL091124. SS is supported by NIH K08 HL096833. We thank Carol J. Bult of the Jackson Laboratory, Bar Harbor, ME for permission to use an expanded unpublished version of the GEO public dataset GSE11539.

\section{Author details}

'Children's Hospital Informatics Program, Boston Children's Hospital, 320 Longwood Avenue, Boston, MA 02115, USA. ${ }^{2}$ Harvard Medical School, Boston, MA, USA. ${ }^{3}$ Channing Division of Network Medicine, Brigham and Women's Hospital, 181 Longwood Avenue, Boston, MA 02115, USA.

${ }^{4}$ Pulmonary Division, Brigham and Women's Hospital, Boston, MA, USA. ${ }^{5}$ Children's Mercy Hospital, Kansas City, MO, USA. ${ }^{6}$ Partners Health Care Center for Personalized Genetic Medicine, Boston, MA, USA. ${ }^{7}$ University of Rochester Medical Center, Rochester, NY, USA. ${ }^{8}$ Channing Division of Network Medicine, Brigham and Women's Hospital and Harvard Medical School, 181 Longwood Avenue, Boston, MA 02115, USA. 'Division of Pediatric Clinical Pharmacology and Medical Toxicology, Children's Mercy Hospital and Clinics, 2401 Gilham Road, Kansas City, MO 64108, USA. ${ }^{10}$ Department of Microbiology and Immunology, University of Rochester Medical Center, 601 Elmwood Avenue, Rochester, NY 14642, USA.

Received: 5 June 2013 Accepted: 31 October 2013

Published: 5 November 2013

\section{References}

1. Masoli M, Fabian D, Holt S, Beasley R: The global burden of asthma: executive summary of the GINA dissemination committee report. Allergy 2004, 59:469-478.

2. Matricardi PM: Prevalence of atopy and asthma in eastern versus western Europe: why the difference? Ann Allergy Asthma Immunol 2001, 87:24-27.

3. Weinberg EG: Urbanization and childhood asthma: an African perspective. J Allergy Clin Immunol 2000, 105:224-231.

4. Platts-Mills TA, Erwin E, Heymann P, Woodfolk J: Is the hygiene hypothesis still a viable explanation for the increased prevalence of asthma? Allergy 2005, 60(Suppl 79):25-31.

5. von Mutius E: The rising trends in asthma and allergic disease. Clin Exp Allergy 1998, 28(Suppl 5):45-49. discussion 50-41.

6. Holick MF: Vitamin D deficiency. N Engl J Med 2007, 357:266-281.

7. Camargo CA Jr, Rifas-Shiman SL, Litonjua AA, Rich-Edwards JW, Weiss ST, Gold DR, Kleinman K, Gillman MW: Maternal intake of vitamin D during pregnancy and risk of recurrent wheeze in children at $3 \mathrm{y}$ of age. Am J Clin Nutr 2007, 85:788-795.

8. Devereux G, Litonjua AA, Turner SW, Craig LC, McNeill G, Martindale S, Helms PJ, Seaton A, Weiss ST: Maternal vitamin D intake during pregnancy and early childhood wheezing. Am J Clin Nutr 2007, 85:853-859.

9. Miyake Y, Sasaki S, Tanaka K, Hirota Y: Dairy food, calcium and vitamin D intake in pregnancy, and wheeze and eczema in infants. Eur Respir $J$ 2010, 35:1228-1234.

10. Nurmatov U, Devereux G, Sheikh A: Nutrients and foods for the primary prevention of asthma and allergy: systematic review and meta-analysis. J Allergy Clin Immunol 2011, 127(724-733):e721-e730.

11. Barker DJ, Martyn CN: The maternal and fetal origins of cardiovascular disease. J Epidemiol Community Health 1992, 46:8-11.

12. Evans KN, Bulmer JN, Kilby MD, Hewison M: Vitamin D and placentaldecidual function. J Soc Gynecol Investig 2004, 11:263-271. 
13. Sundar IK, Rahman I: Vitamin d and susceptibility of chronic lung diseases: role of epigenetics. Front Pharmacol 2011, 2:50.

14. De Luca G, Olivieri F, Melotti G, Aiello G, Lubrano L, Boner AL: Fetal and early postnatal life roots of asthma. J Matern Fetal Neonatal Med 2010, 23(Suppl 3):80-83.

15. Kumar R: Prenatal factors and the development of asthma. Curr Opin Pediatr 2008, 20:682-687.

16. Carbon S, Ireland A, Mungall CJ, Shu S, Marshall B, Lewis S: AmiGO: online access to ontology and annotation data. Bioinformatics 2009, 25:288-289.

17. Ramagopalan SV, Heger A, Berlanga AJ, Maugeri NJ, Lincoln MR, Burrell A, Handunnetthi L, Handel AE, Disanto G, Orton SM, et al: A ChIP-seq defined genome-wide map of vitamin $D$ receptor binding: associations with disease and evolution. Genome Res 2010, 20:1352-1360.

18. Naxerova K, Bult CJ, Peaston A, Fancher K, Knowles BB, Kasif S, Kohane IS: Analysis of gene expression in a developmental context emphasizes distinct biological leitmotifs in human cancers. Genome Biol 2008, 9:R108.

19. Kho AT, Bhattacharya S, Tantisira KG, Carey VJ, Gaedigk R, Leeder JS, Kohane IS, Weiss ST, Mariani TJ: Transcriptomic analysis of human lung development. Am J Respir Crit Care Med 2010, 181:54-63.

20. Dong J, Jiang G, Asmann YW, Tomaszek S, Jen J, Kislinger T, Wigle DA: MicroRNA networks in mouse lung organogenesis. PLoS One 2010 5:e10854.

21. Moffatt MF, Kabesch M, Liang L, Dixon AL, Strachan D, Heath S, Depner M, von Berg A, Bufe A, Rietschel E, et al: Genetic variants regulating ORMDL3 expression contribute to the risk of childhood asthma. Nature 2007 448:470-473.

22. Childhood Asthma Management Program Research Group: The childhood asthma management program (CAMP): design, rationale, and methods. Control Clin Trials 1999, 20:91-120.

23. The Childhood Asthma Management Program Research Group: Long-term effects of budesonide or nedocromil in children with asthma. N Engl J Med 2000, 343:1054-1063.

24. Bosse $Y$, Maghni $K$, Hudson TJ: 1alpha,25-dihydroxy-vitamin D3 stimulation of bronchial smooth muscle cells induces autocrine, contractility, and remodeling processes. Physio/ Genomics 2007, 29:161-168.

25. Irizarry RA, Bolstad BM, Collin F, Cope LM, Hobbs B, Speed TP: Summaries of affymetrix GeneChip probe level data. Nucleic Acids Res 2003, 31:e15.

26. Loader C: Local regression and likelihood. New York: Springer; 1999.

27. Alter O, Brown PO, Botstein D: Generalized singular value decomposition for comparative analysis of genome-scale expression data sets of two different organisms. Proc Natl Acad Sci USA 2003, 100:3351-3356.

28. Kho AT, Bhattacharya S, Mecham BH, Hong J, Kohane IS, Mariani TJ: Expression profiles of the mouse lung identify a molecular signature of time-to-birth. Am J Respir Cell Mol Biol 2009, 40:47-57.

29. Misra J, Schmitt W, Hwang D, Hsiao LL, Gullans S, Stephanopoulos G: Interactive exploration of microarray gene expression patterns in a reduced dimensional space. Genome Res 2002, 12:1112-1120.

30. Huang DW, Sherman BT, Lempicki RA: Systematic and integrative analysis of large gene lists using DAVID bioinformatics resources. Nat Protoc 2009, 4:44-57.

31. Tantisira KG, Lasky-Su J, Harada M, Murphy A, Litonjua AA, Himes BE, Lange C, Lazarus R, Sylvia J, Klanderman B, et al: Genomewide association between GLCCI1 and response to glucocorticoid therapy in asthma. N Engl J Med 2011, 365:1173-1183.

32. Himes BE, Hunninghake GM, Baurley JW, Rafaels NM, Sleiman P, Strachan DP, Wilk JB, Willis-Owen SA, Klanderman B, Lasky-Su J, et al: Genome-wide association analysis identifies PDE4D as an asthma-susceptibility gene. Am J Hum Genet 2009, 84:581-593.

33. Bertram C, Trowern AR, Copin N, Jackson AA, Whorwood CB: The maternal diet during pregnancy programs altered expression of the glucocorticoid receptor and type 211 beta-hydroxysteroid dehydrogenase: potential molecular mechanisms underlying the programming of hypertension in utero. Endocrinology 2001, 142:2841-2853.

34. Mukhopadhyay P, Horn KH, Greene RM, Michele Pisano M: Prenatal exposure to environmental tobacco smoke alters gene expression in the developing murine hippocampus. Reprod Toxicol 2010, 29:164-175.

35. Ogawa T, Rakwal R, Shibato J, Sawa C, Saito T, Murayama A, Kuwagata M, Kageyama H, Yagi M, Satoh K, Shioda S: Seeking gene candidates responsible for developmental origins of health and disease. Congenit Anom (Kyoto) 2011, 51:110-125.
36. Whorwood CB, Firth KM, Budge H, Symonds ME: Maternal undernutrition during early to midgestation programs tissue-specific alterations in the expression of the glucocorticoid receptor, 11 beta-hydroxysteroid dehydrogenase isoforms, and type 1 angiotensin ii receptor in neonatal sheep. Endocrinology 2001, 142:2854-2864.

37. Javaid MK, Crozier SR, Harvey NC, Gale CR, Dennison EM, Boucher BJ, Arden NK, Godfrey KM, Cooper C: Maternal vitamin D status during pregnancy and childhood bone mass at age 9 years: a longitudinal study. Lancet 2006, 367:36-43.

38. Lee KN, Kang HS, Jeon JH, Kim EM, Yoon SR, Song H, Lyu CY, Piao ZH, Kim $\mathrm{SU}, \mathrm{Han} \mathrm{YH}$, et al: VDUP1 is required for the development of natural killer cells. Immunity 2005, 22:195-208.

39. Koh Yl, Shim JU, Wi J, Kwon YE: The role of natural killer T cells in the pathogenesis of acute exacerbation of human asthma. Int Arch Allergy Immunol 2012, 158:131-141.

40. Gao F, Cai SX, Zou F, Li WJ, Zhao HJ: Expression of thioredoxin-binding protein-2/vitamin D3 upregulated protein-1 in peripheral blood eosinophils of asthma patients. Nan Fang Yi Ke Da Xue Xue Bao 2006, 26:371-375.

41. Jin P, Han TH, Ren J, Saunders S, Wang E, Marincola FM, Stroncek DF: Molecular signatures of maturing dendritic cells: implications for testing the quality of dendritic cell therapies. J Trans/ Med 2010, 8:4.

42. Mitsui H, Suarez-Farinas M, Belkin DA, Levenkova N, Fuentes-Duculan J, Coats I, Fujita H, Krueger JG: Combined use of laser capture microdissection and cDNA microarray analysis identifies locally expressed diseaserelated genes in focal regions of psoriasis vulgaris skin lesions. J Invest Dermatol 2012, 132:1615-1626.

43. Vasudevan L, Jeromin A, Volpicelli-Daley L, De Camilli P, Holowka D, Baird B: The beta- and gamma-isoforms of type I PIP5K regulate distinct stages of Ca2+ signaling in mast cells. J Cell Sci 2009, 122:2567-2574.

44. Huynh KK, Eskelinen EL, Scott CC, Malevanets A, Saftig P, Grinstein S: LAMP proteins are required for fusion of lysosomes with phagosomes. EMBO 2007, 26:313-324.

45. Saftig P, Beertsen W, Eskelinen EL: LAMP-2: a control step for phagosome and autophagosome maturation. Autophagy 2008, 4:510-512.

46. $\mathrm{Xu}$ Y, Eissa NT: Autophagy in innate and adaptive immunity. Proc Am Thorac Soc 2010, 7:22-28.

47. Poon AH, Chouiali F, Tse SM, Litonjua AA, Hussain SN, Baglole CJ, Eidelman DH, Olivenstein R, Martin JG, Weiss ST, et al: Genetic and histologic evidence for autophagy in asthma pathogenesis. J Allergy Clin Immunol 2012, 129:569-571.

48. Oettgen HC, Geha RS: IgE in asthma and atopy: cellular and molecular connections. J Clin Invest 1999, 104:829-835.

49. Park CS, Ra DJ, Lee SM, Jeong SW, Uh S, Kim HT, Kim YH: Interleukin-4 and low-affinity receptor for IgE on B cells in peripheral blood of patients with atopic bronchial asthma. J Allergy Clin Immunol 1996, 97:1121-1128.

50. Tsitoura DC, Yeung VP, DeKruyff RH, Umetsu DT: Critical role of B cells in the development of T cell tolerance to aeroallergens. Int Immunol 2002, 14:659-667.

51. Carter KL, Cahir-McFarland E, Kieff E: Epstein-barr virus-induced changes in B-lymphocyte gene expression. J Virol 2002, 76:10427-10436.

doi:10.1186/1755-8794-6-47

Cite this article as: Kho et al:: Vitamin D related genes in lung development and asthma pathogenesis. BMC Medical Genomic 2013 6:47.

\section{Submit your next manuscript to BioMed Central and take full advantage of:}

- Convenient online submission

- Thorough peer review

- No space constraints or color figure charges

- Immediate publication on acceptance

- Inclusion in PubMed, CAS, Scopus and Google Scholar

- Research which is freely available for redistribution 